\title{
Território Territory
}

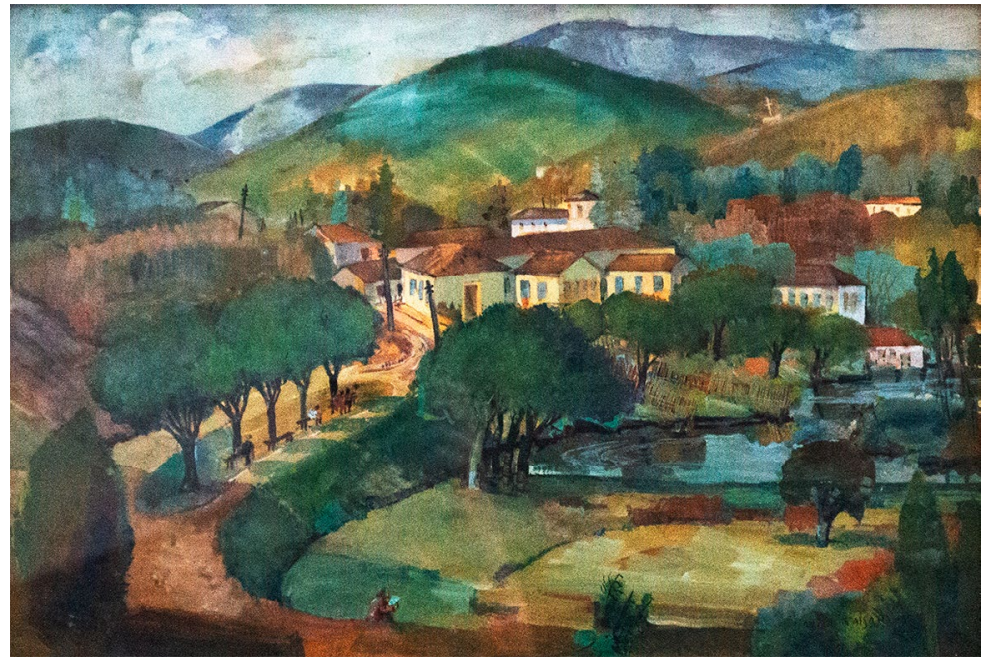

Anita Catarina Malfatti

[Sem título], 1955

Untitled 


\section{Territory}

\section{Paulo Rogério de Freitas Silva}

At first, we established a conversation with Souza (2005, p. 81), to reference territory and its different applications, observing, according to the author, that "The word territory usually evokes the 'national territory' and makes one think of the State-manager par excellence of the national territory,$-[. .]$.$" . The author adds that "However, it need not and should$ not be reduced to that scale or to the association with the State image. Territories exist and are constructed (and deconstructed) in the most diverse scales, [...]."

In this context, we realized that the Brazilian territory, in different scales, was formed over time, based on an exploitation model, through the extraction of redwood, followed by the sugarcane cycle, mining cycle, rubber cycle, coffee cycle, among other not less important circuits, unleashing the genesis of embryonic urban centers that formalized, for example, the urbanization of the territory being evoked.

Those urban centers, distributed over the Brazilian territory, had their geneses established by spontaneous or induced determining processes, the latter being as varied as possible, for, as highlighted by Corrêa (2001, p. 96), "The creation of centers or the development of urban functions along with an economic activity was a general rule in the genesis of the

1 Article originally published in Brazil in Figures, v. 28, 2020 edition.

$2 \mathrm{PhD}$ in Geography from the University of São Paulo (USP). Professor at the Federal University of Alagoas (UFAL), Institute of Geography, Development and Environment (IGDEMA), Postgraduate Program in Geography. Leader of the Research Group: Region, Regionalization and Regionalism, registered in the National Council for Scientific and Technological Development (CNPq). 


\section{Território ${ }^{1}$}

\section{Paulo Rogério de Freitas Silva}

De início, estabelecemos um diálogo com Souza (2005, p. 81), para referenciar território e suas diferentes aplicações, atentando, conforme o autor que, "A palavra território normalmente evoca o 'território nacional' e faz pensar no Estado - gestor por excelência do território nacional -, [...]". O autor acrescenta que, "No entanto, ele não precisa e nem deve ser reduzido a essa escala ou à associação com a figura do Estado. Territórios existem e são construídos (e desconstruídos) nas mais diversas escalas, [...]."

Nesse contexto, constatamos que o território brasileiro, em diferentes escalas, processou-se ao longo do tempo, num modelo de exploração, através da extração do pau-brasil, seguido pelo ciclo da cana-de-açúcar, ciclo da mineração, ciclo da borracha, ciclo do café, entre outros circuitos não menos importantes, desencadeando a gênese de núcleos urbanos embrionários que formalizaram, por exemplo, a urbanização do território em evocação.

Esses núcleos urbanos, distribuídos no território brasileiro, tiveram suas gêneses definidas por processos determinantes espontâneos ou induzidos, sendo estes os mais variados possíveis, pois conforme destaca Corrêa $(2001$, p. 96), "A criação de núcleos ou o desenvolvimento de funções urbanas junto a uma atividade econômica foi regra geral

1 Artigo originalmente publicado no Brasil em Números, v. 28, edição de 2020.

2 Doutor em Geografia pela Universidade de São Paulo (USP). Professor da Universidade Federal de Alagoas (UFAL), Instituto de Geografia, Desenvolvimento e Meio Ambiente (IGDEMA), Programa de Pós-Graduação em Geografia. Líder do Grupo de Pesquisa: Região, Regionalização e Regionalismo, cadastrado no Conselho Nacional de Desenvolvimento Científico e Tecnológico (CNPq). 
Brazilian urban network [...]." The author highlights the creation and development of centers along a sugar mill, a rubber plantation, a mine or a mining, a chapel on a farm, a textile plant, a lodge for donkey troops or in the junction of trading troops. On the other hand, we highlight that the condition that those urban centers reached depended on impulses that tackled them throughout history, fostering a strengthening and conception dynamics of the embryonic centers that advanced, supported by the artifices of each period, or that remained in a perennial lethargic state.

In that scenario, we started from the idea of genetic complexity, based on Corrêa (2001, p. 95), as, in his terms, "The Brazilian urban network comprises a whole set of centers dated from several moments." It is important to observe, through the author's position, that cities created in the first half of the 16th Century, in the beginning of the colonization, and cities born in the 1980s, coexist in the same space, while others are created in the beginning of the 21st Century, in the Amazon, differing in terms of their immediate purposes and agents of creation.

Santos (2005, p. 22) refers to the subject as "generation of cities", when he alludes to the past process of urban creation in Brazil, which was different from urbanization, since that background was subordinate to a natural economy, the relations between places were weak and inconstant, considering that Brazil is a country with such huge territorial dimensions. According to Santos (2005, p. 19), "In the beginning, the 'city' was much more an emanation from a distant power, a will to be present in a remote country."

When dealing with this subject, Santos and Silveira (2008, p. 31) highlight that, in Brazil, "Related to foreign demands, economic zones are formed, as a well as real families and generations of cities, witnessing a sequence of territorial divisions of labor, with different levels of technification."

Nevertheless, according to Santos (2005, p. 21), "[...] it is from the 18th century onwards that urbanization develops and the city home becomes the most important residence of farmers, who only go to their rural properties during sugarcane cutting and milling." It should be highlighted that more than a century was needed until urbanization reached maturity, in the 19th Century, and one more century to acquire the characteristics that we know today.

\section{Regional and territorial overlapping}

We have observed that Brazil was regionalized along the 20th Century, as well as in the first years of the 21 st Century, based on several variables and methodologies, revealing a territorially complex and diverse country. Those regionalizations are developed for statistical purposes, respecting 
na gênese da rede urbana brasileira [...]". O autor destaca a criação e o desenvolvimento de núcleos junto a uma sede de engenho de açúcar, a um seringal, a uma mina ou a um garimpo, a uma capela em fazenda, a uma fábrica têxtil, a uma pousada de tropas de burros ou no entroncamento de tropas de comércio.

Por outro lado, avultamos que a condição que esses núcleos urbanos alcançaram dependeu de impulsos que os acometeram ao longo da história, proporcionando uma dinâmica de fortalecimento e concepção dos núcleos embrionários que obtiveram um avanço, amparados pelos artifícios de cada período ou permaneceram num letargo perene.

Nessa conjuntura, partimos da ideia de complexidade genética, baseados em Corrêa (2001, p. 95), pois, nos termos do autor, "A rede urbana brasileira é constituída por um conjunto de centros datados de diversos momentos." É importante verificar, através do citado autor, que coexistem no mesmo espaço cidades criadas na primeira metade do Século XVI, no início da colonização, e cidades nascidas na década de 1980, enquanto outras mais são criadas no início do Século XXI, na Amazônia, com diferenciação no que se refere aos agentes e propósitos imediatos da criação.

Santos $(2005$, p. 22) se refere ao tema como de "geração de cidades", quando faz alusão ao processo pretérito de criação urbana no Brasil e que se diferenciava de urbanização, pois essa origem estava subordinada a uma economia natural, as relações entre lugares eram fracas e inconstantes, se tratando de um país com tão grandes dimensões territoriais. Inclusive, para Santos (2005, p. 19), "No começo, a 'cidade' era bem mais uma emanação do poder longínquo, uma vontade de marcar presença num país distante".

Santos e Silveira (2008, p. 31), ao se remeterem a esse tema, destacam que no Brasil, "Relacionadas com as demandas do exterior, formam-se zonas econômicas e criam-se verdadeiras famílias e gerações de cidades, testemunhando uma sucessão de divisões territoriais do trabalho, em graus diversos de tecnificação. "

Todavia, para Santos (2005, p. 21), "[...] é a partir do século XVIII que a urbanização se desenvolve e a casa da cidade torna-se a residência mais importante do fazendeiro ou do senhor do engenho, que só vai a sua propriedade rural no momento do corte e da moenda da cana." Destacando que foi necessário mais um século para que a urbanização atingisse sua maturidade, no Século XIX, e mais um século para adquirir as características que conhecemos hoje.

\section{As sobreposições regionais e territoriais}

Conferimos que o Brasil foi regionalizado ao longo do Século XX, assim como nos primeiros anos do Século XXI, baseado em metodologias e variáveis diversas, buscando 
the political-administrative borders and, consequently, respecting the state and municipal borders, according to Map 1.1.

Considering those questions, according to Table 1.1 and Map 1.1, we verified that the Brazilian territory has an absolute total area of 8510820623 km², regionalized into five Geographic Major Regions. They are defined as North, Northeast, Central-West, Southeast and South, and vary in terms of territorial dimension, number and size of states, and, also, in terms of the number of municipalities in each of the respective states, according to Table 1.2. In this context, we highlight the size of the Country and the location of its states, from the Northern Hemisphere, down to the distant lands to the south of the Tropic of Capricorn, i.e., between the headwaters of Uailã River in the far north of Roraima and the Chuí Stream, in Rio Grande do Sul, with a distance of 4378349 km, as well as, from East to West, between Ponta do Seixas, in Paraíba, and the headwaters of Moa River, in Acre, with a distance of $432661 \mathrm{~km}$ between the two extremes, according to Table 1.3 and Map 1.2. It is important to highlight that, in that context of huge territorial dimension, the distances between the state capitals vary in relation to Brasília, according to Table 1.5, being Boa Vista the farthest capital, i.e., 4275 km far, and Goiânia, the closest one, only $209 \mathrm{~km}$ far from the capital of Brazil.

That territorial dimension comprises a multitude of landscapes, arranged from latitudes $+05^{\circ} 16^{\prime} 19^{\prime \prime}$ North and $-33^{\circ} 45^{\prime} 07^{\prime \prime}$ South and longitudes $-34^{\circ} 47^{\prime} 35^{\prime \prime}$ East and $-73^{\circ} 59^{\prime} 26^{\prime \prime}$ West, where the Amazon Forest, Atlantic Forest, Cerrado, Caatinga, Pantanal, Pampa, Araucarian Forest and mangroves prevail. In addition, the distinctive relief pinpoints enclosures in the territory registered as the highest ones in Brazil, according to Table 1.4 , with various toponyms, highlighted by Mount Roraima, in the far north of Brazil, bordering with Venezuela and the Cooperative Republic of Guyana and Cristal Peak, in Minas Gerais. According to Table 1.4, the highest points located in the North and Southeast Major Regions prevail.

\section{Brazilian states and municipalities as several territories}

In this arrangement, we state that the stars laid out in the Brazilian Flag are not uniform and each one represents a Brazilian state plus the Federal District. Their distribution was arranged according to the attributes of the sky in Rio de Janeiro on November 15, 1889, Declaration Day of the Republic of Brazil. The selection of the star corresponding to each state pursues a correlation between the location of the state in the Brazilian territory and the location of the star in the sky, having the State of Alagoas as an example, represented by Theta Scorpii, being Scorpius the constellation and Theta, the star.

Based on that layout, the municipalities that constitute those federation states are the smallest self-governing territorial units, adding up to 5570 
evidenciar um país diverso e complexo territorialmente. Essas regionalizações são elaboradas para fins estatísticos, respeitando os limites político-administrativos, por conseguinte, respeitando os limites estaduais e municipais, conforme Mapa 1.1.

Diante dessas questões, conforme a Tabela 1.1 e Mapa 1.1, conferimos que o território brasileiro tem uma área total absoluta de $8510820623 \mathrm{~km}^{2}$, regionalizado por cinco Macrorregiões Geográficas, definidas como Norte, Nordeste, Centro-Oeste, Sudeste e Sul, que variam de dimensão territorial, de quantidade e tamanho de estados que cada um desses arquétipos contém e, também, pela quantidade de municípios contidos nos respectivos estados, conforme Tabela 1.2. Nesse contexto, avultamos o tamanho do País e a localização dos seus estados desde o Hemisfério Norte, alcançando as terras longínquas ao sul do Trópico de Capricórnio, isto é, entre as nascentes do Rio Uailã no extremo norte de Roraima, e o Arroio Chuí, no Rio Grande do Sul, com uma distância de 4378349 Km, assim como, de Leste a Oeste, entre a Ponta do Seixas, na Paraíba, e a nascente do Rio Moa, no Acre, com um distância de 432661 km entre os dois extremos, conforme se atestam a Tabela 1.3 e o Mapa 1.2. É importante destacar que, nesse contexto de grande dimensão territorial, as distâncias entre as capitais estaduais variam com relação à Brasília, conforme a Tabela 1.5, sendo Boa Vista a capital que está localizada mais distante, isto é, a 4275 Km e Goiânia, a mais próxima, a apenas 209 Km.

Essa dimensão territorial reúne uma diversidade de paisagens, dispostas desde as latitudes $+05^{\circ} 16^{\prime} 19^{\prime \prime}$ Norte e $-33^{\circ} 45^{\prime} 07^{\prime \prime}$ Sul e longitudes $-34^{\circ} 47^{\prime} 35^{\prime \prime}$ Leste e $-73^{\circ} 59^{\prime} 26^{\prime \prime}$ Oeste, formalizada por predominância de Floresta Amazônica, Mata Atlântica, Cerrado, Caatinga, Pantanal, campos sulinos, mata de araucárias e mangues. Além disso, o relevo diferenciado assinala recintos no território cadastrados como os mais altos do País, conforme apresentado na Tabela 1.4, com topônimos variados, com destaque aqui para o Monte Roraima, extremos norte do Brasil, fronteira com a Venezuela e com a República Cooperativa da Guiana e o Pico do Cristal, em Minas Gerais, onde conferimos, segundo a Tabela 1.4, que ocorre uma predominância dos pontos mais altos localizados nas Macrorregiões Norte e Sudeste do Brasil.

\section{Os Estados e os Municípios brasileiros como territórios diversos}

Nesse arranjo em foco, discorremos que as estrelas dispostas na Bandeira do Brasil não são uniformes e cada uma representa um estado brasileiro mais o Distrito Federal. A distribuição das mesmas foi arranjada a partir dos atributos do céu do Rio de Janeiro, no dia 15 de novembro de 1889, dia da Proclamação da República do Brasil e a escolha da estrela correspondente de cada estado procura seguir uma correlação entre a localização do estado no território brasileiro e a paragem da estrela no céu, tendo como 
distributed along the Brazilian territory, being 450 located in the North Major Region; 1794 in the Northeast Major Region; 467 in the CentralWest Major Region; 1668 in the Southeast Major Region; and 1191 in the South Major Region (Table 1.2).

It is important to stress that the dynamics of the political emancipation in Brazil varied in different periods, since in the 1940s the Country concentrated 1574 municipalities; in 1970, it reached 3 952; in 1990, 4491; reaching 5507 in the year of 2000; and 5 570, in 2019.

In these circumstances, we realized that the process of colonization and appropriation of the Brazilian territory also expressed itself by means of the political-administrative division in municipalities, many of which have been created throughout history, though in a changing pace. Also, that some outstanding episodes stand out as if some important periods of the political life correspond to a wave of creation of municipalities.

The North Major Region concentrates the states with the largest territorial dimensions in Brazil, Amazonas and Pará, the equivalent to $18.32 \%$ and $14.64 \%$, respectively, of the Country, concentrating 62 and 144 municipalities, respectively. Roraima is equivalent to $2.64 \%$ of the National Territory, being the less populous state for concentrating 618210 inhabitants and the least populated in Brazil, with a population density of 2.76 inhabitants per square kilometer, distributed in a territory of $224273831 \mathrm{~km}^{2}$.Comprising 15 municipalities, it is the state with the smallest number of municipalities in Brazil, being the territorial dimension of some of them larger than some federation states. Amapá should also be highlighted. It comprises 16 municipalities, concentrating 860063 inhabitants and ranked as the second less populous in Brazil.

Roraima and Amapá have most of their territories located in the Northern Hemisphere, among the Guyanas, being real spearheads towards the north of South America.

As to the Northeast Major Region, it concentrates the largest number of municipalities, adding up to 1794 , as well as states with the smallest territorial dimensions in Brazil, such as Sergipe and Alagoas, which have, respectively, 21926908 km$^{2}$ and 27843295 km², with Alagoas concentrating 102 municipalities, and Sergipe, 75 municipalities.

The most populous states in Brazil - São Paulo, Rio de Janeiro and Minas Gerais - are located in the Southeast Major Region, highlighting the largest metropolitan areas, economic centers of the Country. Minas Gerais is the Brazilian state with the largest number of municipalities, adding up to 853 territories, the equivalent to $15.5 \%$ of the total number of municipalities in the National Territory.

The South Major Region concentrates three states, comprising 1191 municipalities. However, the region concentrated only 181 territories in 
exemplo o Estado de Alagoas que é representado pela Estrela Teta de Escorpião, sendo Escorpião, a constelação, e Teta, a estrela.

A partir dessa disposição, os municípios que compõem esses estados da federação, são as menores unidades territoriais com governo próprio, totalizando 5570 distribuídos no território brasileiro, estando, 450 localizados na Macrorregião Norte; 1794 na Macrorregião Nordeste; 467 na Macrorregião Centro-Oeste; 1668 na Macrorregião Sudeste; e 1191 na Macrorregião Sul (Tabela 1.2).

É importante frisar que a dinâmica das emancipações políticas no Brasil variou em períodos diversos, pois na década de 1940 o País concentrava 1574 municípios; em 1970, passou a concentrar 3 952; em 1990, 4 491; alcançando 5507 no ano de 2000; e 5 570, em 2019.

Nessas circunstâncias, atestamos que o processo de colonização e apropriação do território brasileiro se expressou também pela divisão política-administrativa em municípios, que muitos destes têm sido criados ao longo de toda a história, mas em ritmo variável, e que alguns episódios notáveis se destacam como se alguns períodos importantes da vida política correspondessem a uma onda de criação de municípios.

A Macrorregião Norte concentra os estados com as maiores dimensões territoriais do Brasil, o Amazonas e o Pará, equivalendo respectivamente a 18,32\% e $14,64 \%$ do País, concentrando 62 e 144 municípios, respectivamente. Roraima, equivale a 2,64\% do Território Nacional, sendo o estado menos populoso por concentrar 618210 habitantes e menos povoado do Brasil, com uma densidade demográfica de 2,76 habitantes por quilômetro quadrado, distribuídos num território de 224273831 Km². Com 15 municípios é o estado com a menor quantidade de municipalidades do Brasil, tendo alguns com dimensão territorial maior que alguns estados da federação. Merece ênfase também o Amapá que reúne 16 municípios, concentrando 860063 habitantes, classificado como o segundo menos populoso do Brasil.

Roraima e Amapá, têm a maior parte de seus territórios localizados no Hemisfério Norte, situados entre as Guianas, sendo verdadeiras pontas de lança em direção ao norte da América do Sul.

No que se refere à Macrorregião Nordeste, esta região concentra a maior quantidade de municípios, somando, 1794 , assim como estados com as menores dimensões territoriais do País, a exemplo de Sergipe e de Alagoas, que possuem respectivamente, $21926908 \mathrm{Km}^{2}$ e $27843295 \mathrm{Km}^{2}$, com Alagoas, concentrando 102 municípios, e Sergipe 75 municípios. 
1940, highlighted by Rio Grande do Sul, which changed from 88 territories, in 1940, to 497 municipalities, distributed along the last frontier.

In the Central-West Major Region, the State of Mato Grosso stands out in terms of territorial dimension, with 903206997 km², being the third largest Brazilian state, concentrating 79 municipalities. The Federal District stands out in that arrangement, due to the importance of the city of Brasília, the political center of the Country.

\section{Complementary remarks}

The Brazilian political-administrative organization comprises the Union, the States, the Federal District and the Municipalities, with the possible occurrence of sub-divisions, formation of new ones, creation, incorporation, mergers and break-ups of states and municipalities (BRASIL, 1990). However, Santos (2005, p. 29), in a reference to that type of arrangement, stresses that "For many centuries, Brazil was a large archipelago comprising sub-spaces that evolved according to their own logic, [...]." The author adds that, "Those, however, had a minimal relation with each other, not being interdependent."

\section{References}

BRASIL. Constituição (1988). Constituição da República Federativa do Brasil: promulgada em 5 de outubro de 1988. 4. Ed. São Paulo: Saraiva, 1990.

CORREA, R. L. Trajetórias geográficas. 2. Ed. Rio de Janeiro: Bertrand Brasil, 2001. 302 p.

SANTOS, M. A urbanização brasileira. 5. Ed. São Paulo: EdUSP, 2005. 174 p. (Coleção Milton Santos, 6).

SANTOS, M.; SILVEIRA, M. L. O Brasil: território e sociedade no início do século XXI. 12. Ed. Rio de Janeiro: Record, 2008.

SOUZA, M. L. de. O território: sobre espaço e poder, autonomia e desenvolvimento. In: CASTRO, I. E. de; GOMES, P. C. da C.; CORRÊA, R. L. (Org.). Geografia: conceitos e temas. Rio de Janeiro: Bertrand Brasil, 2005.

Translated by: La-Fayette Côrtes Neto 
Na Macrorregião Sudeste, localizam-se os estados mais populosos do Brasil, São Paulo, Rio de Janeiro e Minas Gerais, se destacando as maiores regiões metropolitanas, centros de gestão econômica do País. Minas Gerais é o estado brasileiro com a maior quantidade de municípios totalizando 853 territórios que equivalem a 15,5\% do total de municípios do Território Nacional.

Na Macrorregião Sul, concentram-se três estados, reunindo 1191 municípios, contudo no ano de 1940, a região concentrava apenas 181 territórios, tendo destaque o Rio Grande do Sul que de 88 territórios, em 1940, passou a concentrar 497 municípios, distribuídos na última fronteira.

Na Macrorregião Centro-Oeste, destaca-se, em termos de dimensão territorial, o Estado de Mato Grosso com $903206997 \mathrm{Km}^{2}$, sendo o terceiro maior estado do Brasil, concentrando 79 municípios. Destaca-se nesse arranjo o Distrito Federal, pela importância da cidade de Brasília, centro de gestão política do País.

\section{Complementando}

A organização político-administrativa do Brasil compreende a União, os Estados, o Distrito Federal e os Municípios, destacando-se que pode acontecer subdivisão, formação de novos, criação, incorporação, fusão e desmembramentos dos estados e dos municípios (BRASIL, 1990). Porém, Santos (2005, p. 29), ao fazer referência a esse tipo de arranjo, ressalta que "O Brasil foi, durante muitos séculos, um grande arquipélago, formado por subespaços que evoluíam segundo logicas próprias, [...]". O autor adiciona que, "Estes, porém, tinham entre si escassa relação, não sendo interdependentes."

\section{Referências}

BRASIL. Constituição (1988). Constituição da República Federativa do Brasil:promulgada em 5 de outubro de 1988. 4. ed. São Paulo: Saraiva, 1990.

CORRÊA, R. L. Trajetórias geográficas. 2. ed. Rio de Janeiro: Bertrand Brasil, 2001. 302 p.

SANTOS, M. A urbanização brasileira. 5. ed. São Paulo: EdUSP, 2005. 174 p. (Coleção Milton Santos, 6).

SANTOS, M.; SILVEIRA, M. L. O Brasil: território e sociedade no início do século XXI. 12. ed. Rio de Janeiro: Record, 2008.

SOUZA, M. L. de. O território: sobre espaço e poder, autonomia e desenvolvimento. In: CASTRO, I. E. de; GOMES, P. C. da C.; CORRÊA, R. L. (Org.). Geografia: conceitos e temas. Rio de Janeiro: Bertrand Brasil, 2005. 
Tabela 1.1 - Área total do País - 2019

Table 1.1 - Total area of Brazil - 2019

(continua/to be continued)

\begin{tabular}{|c|c|c|c|}
\hline \multirow{3}{*}{$\begin{array}{c}\text { Grandes Regiões } \\
\text { e } \\
\text { Unidades da Federação/ } \\
\text { Major Regions and Federation Units }\end{array}$} & \multicolumn{3}{|c|}{$\begin{array}{c}\text { Área total/ } \\
\text { Total area }\end{array}$} \\
\hline & \multirow{2}{*}{$\begin{array}{c}\text { Absoluta } \\
\left(\mathrm{km}^{2}\right) / \\
\text { Absolute } \\
\left(\mathrm{km}^{2}\right)\end{array}$} & \multicolumn{2}{|c|}{$\begin{array}{c}\text { Relativa (\%)/ } \\
\text { Relative (\%) }\end{array}$} \\
\hline & & $\begin{array}{l}\text { Brasil/ } \\
\text { Brazil }\end{array}$ & $\begin{array}{l}\text { Regiões/ } \\
\text { Regions }\end{array}$ \\
\hline Brasil/ Brazil & 8510820,623 & 100,00 & .. \\
\hline Norte/North & 3851281,390 & 45,25 & 100,00 \\
\hline Rondônia & 237765,233 & 2,79 & 6,17 \\
\hline Acre & 164123,738 & 1,93 & 4,26 \\
\hline Amazonas & 1559168,117 & 18,32 & 40,48 \\
\hline Roraima & 224273,831 & 2,64 & 5,82 \\
\hline Pará & 1245759,305 & 14,64 & 32,35 \\
\hline Amapá & 142470,762 & 1,67 & 3,70 \\
\hline Tocantins & 277720,404 & 3,26 & 7,21 \\
\hline Nordeste/Northeast & 1551991,426 & 18,24 & 100,00 \\
\hline Maranhão & 329642,170 & 3,87 & 21,24 \\
\hline Piauí & 251616,823 & 2,96 & 16,21 \\
\hline Ceará & 148894,757 & 1,75 & 9,59 \\
\hline Rio Grande do Norte & 52809,602 & 0,62 & 3,40 \\
\hline Paraíba & 56467,239 & 0,66 & 3,64 \\
\hline Pernambuco & 98068,021 & 1,15 & 6,32 \\
\hline Alagoas & 27843,295 & 0,33 & 1,79 \\
\hline Sergipe & 21926,908 & 0,26 & 1,41 \\
\hline Bahia & 564722,611 & 6,64 & 36,39 \\
\hline Sudeste/Southeast & 924565,469 & 10,86 & 100,00 \\
\hline Minas Gerais & 586521,121 & 6,89 & 63,44 \\
\hline Espírito Santo & 46074,444 & 0,54 & 4,98 \\
\hline Rio de Janeiro & 43750,423 & 0,51 & 4,73 \\
\hline São Paulo & 248219,481 & 2,92 & 26,85 \\
\hline Sul/South & 576743,308 & 6,78 & 100,00 \\
\hline Paraná & 199305,236 & 2,34 & 34,56 \\
\hline Santa Catarina & 95730,921 & 1,12 & 16,60 \\
\hline Rio Grande do Sul & 281707,151 & 3,31 & 48,84 \\
\hline
\end{tabular}


Tabela 1.1 - Área total do País - 2019

Table 1.1 - Total area of Brazil - 2019

(conclusão/concluded)

\begin{tabular}{|c|c|c|c|}
\hline \multirow{3}{*}{$\begin{array}{c}\text { Grandes Regiões } \\
\text { e } \\
\text { Unidades da Federação/ } \\
\text { Major Regions and Federation Units }\end{array}$} & \multicolumn{3}{|c|}{$\begin{array}{c}\text { Área total/ } \\
\text { Total area }\end{array}$} \\
\hline & \multirow{2}{*}{$\begin{array}{l}\text { Absoluta } \\
\left(\mathrm{km}^{2}\right) / \\
\text { Absolute } \\
\left(\mathrm{km}^{2}\right)\end{array}$} & \multicolumn{2}{|c|}{$\begin{array}{l}\text { Relativa (\%)/ } \\
\text { Relative (\%) }\end{array}$} \\
\hline & & $\begin{array}{l}\text { Brasil/ } \\
\text { Brazil }\end{array}$ & $\begin{array}{l}\text { Regiões/ } \\
\text { Regions }\end{array}$ \\
\hline Centro-Oeste/Central-West & 1606239,030 & 18,87 & 100,00 \\
\hline Mato Grosso do Sul & 357145,535 & 4,20 & 22,23 \\
\hline Mato Grosso & 903206,997 & 10,61 & 56,23 \\
\hline Goiás & 340125,715 & 4,00 & 21,18 \\
\hline Distrito Federal/Federal District & 5760,783 & 0,07 & 0,36 \\
\hline
\end{tabular}

Fonte/Source: IBGE, Diretoria de Geociências, Coordenação de Estruturas Territoriais, Malha Municipal 2018.

\section{Mapa 1.1 - Mapa político do Brasil Map 1.1 - Political map of Brazil}

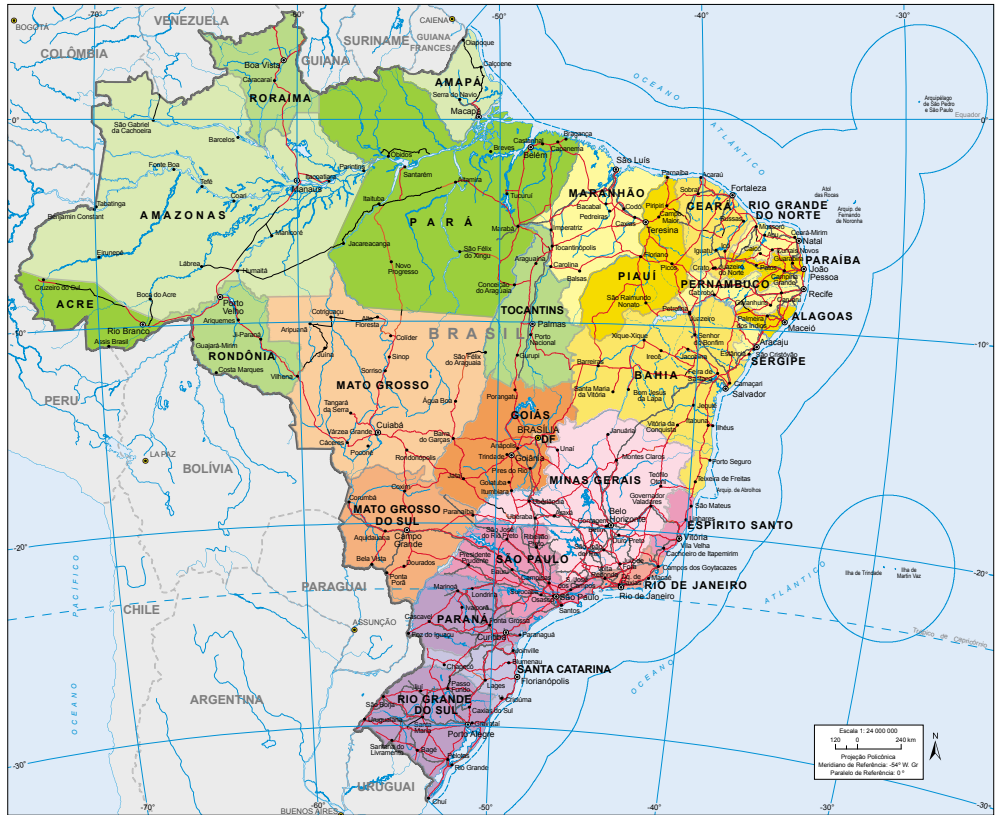

Fonte/Source: IBGE, Diretoria de Geociências. 
Tabela 1.2 - Evolução político-administrativa do País - 1940/2019

Table 1.2 - Administrative evolution of Brazil-1940/2019

\begin{tabular}{|c|c|c|c|c|c|c|c|}
\hline \multirow{2}{*}{$\begin{array}{l}\text { Grandes Regiões e } \\
\text { Unidades da Federação/ } \\
\text { Major Regions and } \\
\text { Federation Units }\end{array}$} & \multicolumn{7}{|c|}{$\begin{array}{l}\text { Municípios criados e instalados (Até 01.09)/ } \\
\text { Municipalities created and installed (Until Sep 1st) }\end{array}$} \\
\hline & $1940(1)$ & 1950 & 1960 & 1970 & 1980 & 1990 & 2000 \\
\hline Brasil/Brazil & 1574 & 1889 & 2766 & 3952 & 3974 & 4491 & 5507 \\
\hline Norte/North & 88 & 99 & 120 & 143 & 153 & 298 & 449 \\
\hline Rondônia & - & 2 & 2 & 2 & 7 & 23 & 52 \\
\hline Acre & 7 & 7 & 7 & 7 & 12 & 12 & 22 \\
\hline Amazonas & 28 & 25 & 44 & 44 & 44 & 62 & 62 \\
\hline Roraima & - & 2 & 2 & 2 & 2 & 8 & 15 \\
\hline Pará & 53 & 59 & 60 & 83 & 83 & 105 & 143 \\
\hline Amapá & - & 4 & 5 & 5 & 5 & 9 & 16 \\
\hline Tocantins & - & - & - & - & - & 79 & 139 \\
\hline Nordeste/Northeast & 584 & 609 & 903 & 1376 & 1375 & 1509 & 1787 \\
\hline Maranhão & 65 & 72 & 91 & 130 & 130 & 136 & 217 \\
\hline Piauí & 47 & 49 & 71 & 114 & 114 & 118 & 221 \\
\hline Ceará & 79 & 79 & 142 & 142 & 141 & 178 & 184 \\
\hline Rio Grande do Norte & 42 & 48 & 83 & 150 & 150 & 152 & 166 \\
\hline Paraíba & 41 & 41 & 88 & 171 & 171 & 171 & 223 \\
\hline Pernambuco & 85 & 91 & 103 & 165 & 165 & (2) 168 & (2) 185 \\
\hline Alagoas & 33 & 37 & 69 & 94 & 94 & 97 & 101 \\
\hline Sergipe & 42 & 42 & 62 & 74 & 74 & 74 & 75 \\
\hline Bahia & 150 & 150 & 194 & 336 & 336 & 415 & 415 \\
\hline Sudeste/Southeast & 641 & 845 & 1085 & 1410 & 1410 & 1432 & 1666 \\
\hline Minas Gerais & 288 & 386 & 483 & 722 & 722 & 723 & 853 \\
\hline Espírito Santo & 32 & 33 & 37 & 53 & 53 & 67 & 77 \\
\hline Rio de Janeiro & 51 & 57 & 62 & 64 & 64 & 70 & 91 \\
\hline São Paulo & 270 & 369 & 503 & 571 & 571 & 572 & 645 \\
\hline Sul/South & 181 & 224 & 414 & 717 & 719 & 873 & 1159 \\
\hline Paraná & 49 & 80 & 162 & 288 & 290 & 323 & 399 \\
\hline Santa Catarina & 44 & 52 & 102 & 197 & 197 & 217 & 293 \\
\hline Rio Grande do Sul & 88 & 92 & 150 & 232 & 232 & 333 & 467 \\
\hline Centro-Oeste/Central-West & 80 & 112 & 244 & 306 & 317 & 379 & 446 \\
\hline Mato Grosso do Sul & - & - & - & - & 55 & 72 & 77 \\
\hline Mato Grosso & 28 & 35 & 64 & 84 & 38 & 95 & 126 \\
\hline Goiás & 52 & 77 & 179 & 221 & 223 & 211 & 242 \\
\hline Distrito Federal/Federal District & - & - & 1 & 1 & 1 & 1 & 1 \\
\hline
\end{tabular}


Tabela 1.2 - Evolução político-administrativa do País - 1940/2019

Table 1.2 - Administrative evolution of Brazil - 1940/2019

\begin{tabular}{|c|c|c|c|c|c|c|c|c|}
\hline \multirow{2}{*}{$\begin{array}{l}\text { Grandes Regiões e } \\
\text { Unidades da Federação/ } \\
\text { Major Regions and } \\
\text { Federation Units }\end{array}$} & \multicolumn{8}{|c|}{$\begin{array}{l}\text { Municípios criados e instalados (Até 01.09)/ } \\
\text { Municipalities created and installed (Until Sep 1st) }\end{array}$} \\
\hline & 2010 & 2013 & 2014 & 2015 & 2016 & 2017 & 2018 & 2019 \\
\hline Brasil/Brazil & 5565 & 5570 & 5570 & 5570 & 5570 & 5570 & 5570 & 5570 \\
\hline Norte/North & 449 & 450 & 450 & 450 & 450 & 450 & 450 & 450 \\
\hline Rondônia & 52 & 52 & 52 & 52 & 52 & 52 & 52 & 52 \\
\hline Acre & 22 & 22 & 22 & 22 & 22 & 22 & 22 & 22 \\
\hline Amazonas & 62 & 62 & 62 & 62 & 62 & 62 & 62 & 62 \\
\hline Roraima & 15 & 15 & 15 & 15 & 15 & 15 & 15 & 15 \\
\hline Pará & 143 & 144 & 144 & 144 & 144 & 144 & 144 & 144 \\
\hline Amapá & 16 & 16 & 16 & 16 & 16 & 16 & 16 & 16 \\
\hline Tocantins & 139 & 139 & 139 & 139 & 139 & 139 & 139 & 139 \\
\hline Nordeste/Northeast & 1794 & 1794 & 1794 & 1794 & 1794 & 1794 & 1794 & 1794 \\
\hline Maranhão & 217 & 217 & 217 & 217 & 217 & 217 & 217 & 217 \\
\hline Piauí & 224 & 224 & 224 & 224 & 224 & 224 & 224 & 224 \\
\hline Ceará & 184 & 184 & 184 & 184 & 184 & 184 & 184 & 184 \\
\hline Rio Grande do Norte & 167 & 167 & 167 & 167 & 167 & 167 & 167 & 167 \\
\hline Paraíba & 223 & 223 & 223 & 223 & 223 & 223 & 223 & 223 \\
\hline Pernambuco & (2) 185 & (2) 185 & (2) 185 & (2) 185 & (2) 185 & (2) 185 & (2) 185 & (2) 185 \\
\hline Alagoas & 102 & 102 & 102 & 102 & 102 & 102 & 102 & 102 \\
\hline Sergipe & 75 & 75 & 75 & 75 & 75 & 75 & 75 & 75 \\
\hline Bahia & 417 & 417 & 417 & 417 & 417 & 417 & 417 & 417 \\
\hline Sudeste/Southeast & 1668 & 1668 & 1668 & 1668 & 1668 & 1668 & 1668 & 1668 \\
\hline Minas Gerais & 853 & 853 & 853 & 853 & 853 & 853 & 853 & 853 \\
\hline Espírito Santo & 78 & 78 & 78 & 78 & 78 & 78 & 78 & 78 \\
\hline Rio de Janeiro & 92 & 92 & 92 & 92 & 92 & 92 & 92 & 92 \\
\hline São Paulo & 645 & 645 & 645 & 645 & 645 & 645 & 645 & 645 \\
\hline Sul/South & 1188 & 1191 & 1191 & 1191 & 1191 & 1191 & 1191 & 1191 \\
\hline Paraná & 399 & 399 & 399 & 399 & 399 & 399 & 399 & 399 \\
\hline Santa Catarina & 293 & 295 & 295 & 295 & 295 & 295 & 295 & 295 \\
\hline Rio Grande do Sul & 496 & 497 & 497 & 497 & 497 & 497 & 497 & 497 \\
\hline Centro-Oeste/Central-West & 466 & 467 & 467 & 467 & 467 & 467 & 467 & 467 \\
\hline Mato Grosso do Sul & 78 & 79 & 79 & 79 & 79 & 79 & 79 & 79 \\
\hline Mato Grosso & 141 & 141 & 141 & 141 & 141 & 141 & 141 & 141 \\
\hline Goiás & 246 & 246 & 246 & 246 & 246 & 246 & 246 & 246 \\
\hline Distrito Federal/Federal District & 1 & 1 & 1 & 1 & 1 & 1 & 1 & 1 \\
\hline
\end{tabular}

Fonte/Source: IBGE, Diretoria de Geociências, Coordenação de Estruturas Territoriais, Banco de Estruturas Territoriais 2018.

(1) Unidades administrativas em 01/09/2019. (2) Inclui o Distrito Estadual de Fernando de Noronha.

(1) Administrative units on 2019 July 1st. (2) Includes the State District of Fernando de Noronha. 
Tabela 1.3 - Pontos extremos do País e suas distâncias - 2019

Table 1.3 -Extreme points of Brazil and their distances - 2019

\begin{tabular}{|c|c|c|c|c|}
\hline \multirow{2}{*}{$\begin{array}{l}\text { Extremo/ } \\
\text { Extreme } \\
\text { points }\end{array}$} & \multicolumn{2}{|c|}{$\begin{array}{l}\text { Coordenadas geográficas/ } \\
\text { Geographic coordinates }\end{array}$} & \multirow{2}{*}{$\begin{array}{l}\text { Localização/ } \\
\text { Location }\end{array}$} & \multirow{2}{*}{$\begin{array}{l}\text { Distância } \\
(\mathrm{km}) / \\
\text { Distance } \\
(\mathrm{km})\end{array}$} \\
\hline & $\begin{array}{l}\text { Latitude/ } \\
\text { Latitude }\end{array}$ & $\begin{array}{l}\text { Longitude/ } \\
\text { Longitude }\end{array}$ & & \\
\hline $\begin{array}{l}\text { Norte/ } \\
\text { North }\end{array}$ & $+05^{\circ} 16^{\prime} 19^{\prime \prime}$ & $-60^{\circ} 12^{\prime} 45^{\prime \prime}$ & $\begin{array}{l}\text { Nascente do rio Ailã (Uiramutã - RR)/ } \\
\text { Source of Ailã river (Uiramutã - RR) }\end{array}$ & \\
\hline $\begin{array}{l}\text { Sul/ } \\
\text { South }\end{array}$ & $-33^{\circ} 45^{\prime} 07^{\prime \prime}$ & $-53^{\circ} 23^{\prime} 50^{\prime \prime}$ & $\begin{array}{l}\text { Arroio Chuí (Santa Vitória } \\
\text { do Palmar - RS)/Chuí Brook } \\
\text { (Santa Vitória do Palmar-RS) }\end{array}$ & - 4378,349 \\
\hline $\begin{array}{l}\text { Leste/ } \\
\text { East }\end{array}$ & $-07^{\circ} 09^{\prime} 18^{\prime \prime}$ & $-34^{\circ} 47^{\prime} 35^{\prime \prime}$ & $\begin{array}{l}\text { Ponta do Seixas (Cabo Branco/ } \\
\text { João Pessoa - PB)/ } \\
\text { Point of Seixas (Cape Branco/ } \\
\text { oão Pessoa-PB) }\end{array}$ & \\
\hline $\begin{array}{l}\text { Oeste/ } \\
\text { West }\end{array}$ & $-07^{\circ} 32^{\prime} 09^{\prime \prime}$ & $-73^{\circ} 59^{\prime} 26^{\prime \prime}$ & $\begin{array}{l}\text { Nascente do rio Moa (Mâncio } \\
\text { Lima - } A C \text { )/ } \\
\text { Source of Moa river (Mâncio Lima - } A C \text { ) }\end{array}$ & \\
\hline
\end{tabular}

Fonte/Source: IBGE, Diretoria de Geociências, Coordenação de Estruturas Territoriais, Malha Municipal 2018.

Nota: Coordenadas Geográficas no datum SIRGAS2000, com distâncias em linha reta obtidas através do modelo elipsoidal./Note: Geographic Coordinates in datum SIRGAS2000 with distances in a straight line obtained from the ellipsoidal model. 
Mapa 1.2 - Pontos extremos e pontos mais altos do País Map 1.2 - Extreme points and highest points in Brazil

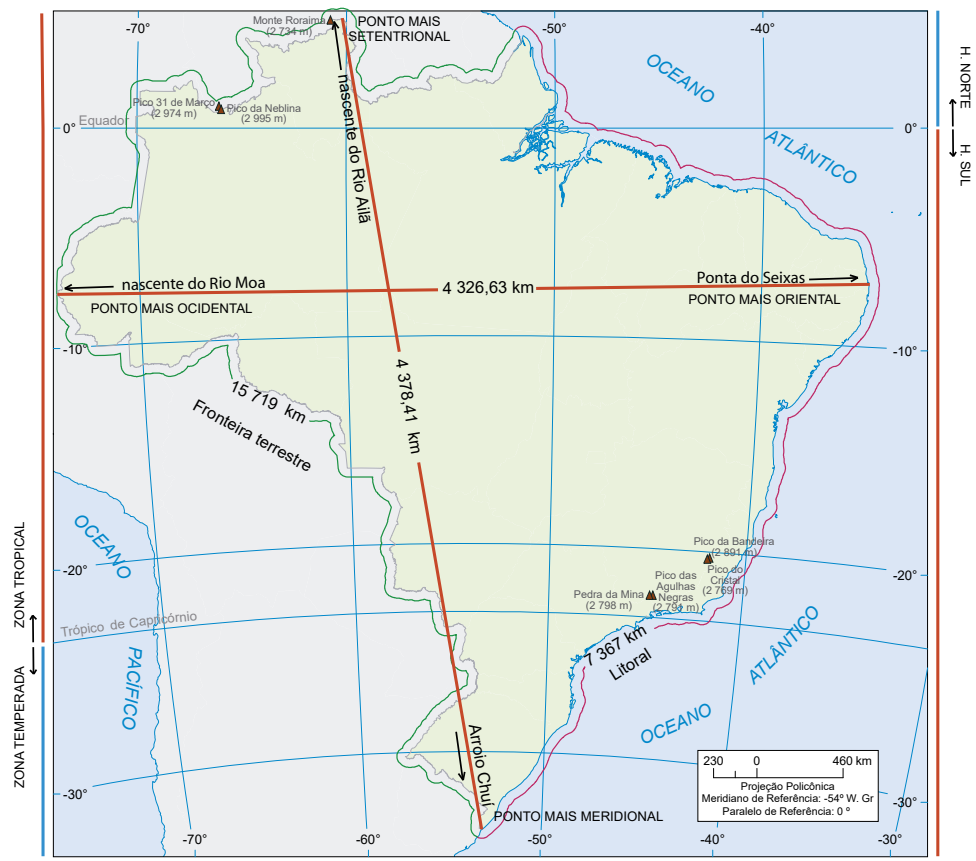

Fonte/Source: IBGE, Diretoria de Geociências. 
Tabela 1.4 - Pontos mais altos do País - 2020

Table 1.4 - Highest points in Brazil - 2020

\begin{tabular}{|c|c|c|c|}
\hline $\begin{array}{l}\text { Topônimos/ } \\
\text { Toponyms }\end{array}$ & $\begin{array}{l}\text { Unidades da Federação/ } \\
\text { Federative Units }\end{array}$ & $\begin{array}{l}\text { Localização/ } \\
\text { Location }\end{array}$ & $\begin{array}{l}\text { Altitude }(\mathrm{m}) / \\
\text { Altitude }(m)\end{array}$ \\
\hline $\begin{array}{l}\text { Pico da Neblina (1) / } \\
\text { Neblina Peak (1) }\end{array}$ & Amazonas & $\begin{array}{l}\text { Serra Imeri/ } \\
\text { Imeri Range }\end{array}$ & 2995,3 \\
\hline $\begin{array}{l}\text { Pico } 31 \text { de Março (1) / } \\
31 \text { de Março Peak (1) }\end{array}$ & Amazonas (2) & $\begin{array}{l}\text { Serra Imeri/ } \\
\text { Imeri Range }\end{array}$ & 2974,2 \\
\hline $\begin{array}{l}\text { Pico da Bandeira (1) / } \\
\text { Bandeira Peak (1) }\end{array}$ & Minas Gerais/Espírito Santo & $\begin{array}{l}\text { Serra do Caparaó/ } \\
\text { Caparaó Range }\end{array}$ & 2891,3 \\
\hline $\begin{array}{l}\text { Pedra da Mina (1) / } \\
\text { Mina Rock (1) }\end{array}$ & Minas Gerais/São Paulo & $\begin{array}{l}\text { Serra da Mantiqueira/ } \\
\text { Mantiqueira Range }\end{array}$ & 2798,1 \\
\hline $\begin{array}{l}\text { Pico das Agulhas Negras (1)/ } \\
\text { Agulhas Negras Peak (1) }\end{array}$ & Minas Gerais/Rio de Janeiro & $\begin{array}{l}\text { Serra do Itatiaia/ } \\
\text { Itatiaia Range }\end{array}$ & 2790,9 \\
\hline $\begin{array}{l}\text { Pico do Cristal (1) / } \\
\text { Cristal Peak (1) }\end{array}$ & Minas Gerais & $\begin{array}{l}\text { Serra do Caparaó/ } \\
\text { Caparaó Range }\end{array}$ & 2769,1 \\
\hline $\begin{array}{l}\text { Monte Roraima (1) / } \\
\text { Roraima Mount (1) }\end{array}$ & Roraima (2) (3) & $\begin{array}{l}\text { Serra do Pacaraima/ } \\
\text { Pacaraima Range }\end{array}$ & 2734,1 \\
\hline $\begin{array}{l}\text { Morro do Couto (4) / } \\
\text { Couto Mount (4) }\end{array}$ & Rio de Janeiro & $\begin{array}{l}\text { Serra das Prateleiras/ } \\
\text { Prateleiras Range }\end{array}$ & 2687,0 \\
\hline $\begin{array}{l}\text { Pedra do Sino de Itatiaia/ } \\
\text { Sino de Itatiaia Rock }\end{array}$ & Minas Gerais & $\begin{array}{l}\text { Serra da Mantiqueira/ } \\
\text { Mantiqueira Range }\end{array}$ & 2670,0 \\
\hline $\begin{array}{l}\text { Pico dos Três Estados/ } \\
\text { Três Estados Peak }\end{array}$ & $\begin{array}{l}\text { São Paulo/Minas Gerais/ } \\
\text { Rio de Janeiro }\end{array}$ & $\begin{array}{l}\text { Serra da Mantiqueira/ } \\
\text { Mantiqueira Range }\end{array}$ & 2665,0 \\
\hline $\begin{array}{l}\text { Pedra do Altar (4) / } \\
\text { Altar Rock (4) }\end{array}$ & Rio de Janeiro & $\begin{array}{l}\text { Serra da Mantiqueira/ } \\
\text { Mantiqueira Range }\end{array}$ & 2663,0 \\
\hline $\begin{array}{l}\text { Morro da Cruz do Negro / } \\
\text { Cruz do Negro Mount }\end{array}$ & Espírito Santo & $\begin{array}{l}\text { Serra do Caparaó/ } \\
\text { Caparaó Range }\end{array}$ & 2658,0 \\
\hline $\begin{array}{l}\text { Pedra Roxa/ } \\
\text { Roxa Rock }\end{array}$ & Espírito Santo & $\begin{array}{l}\text { Serra do Caparaó/ } \\
\text { Caparaó Range }\end{array}$ & 2649,0 \\
\hline $\begin{array}{l}\text { Pico do Tesouro/ } \\
\text { Tesouro Peak }\end{array}$ & Espírito Santo & $\begin{array}{l}\text { Serra do Caparaó/ } \\
\text { Caparaó Range }\end{array}$ & 2620,0 \\
\hline $\begin{array}{l}\text { Pico da Maromba (4) / } \\
\text { Maromba Peak (4) }\end{array}$ & Rio de Janeiro & $\begin{array}{l}\text { Serra da Mantiqueira/ } \\
\text { Mantiqueira Range }\end{array}$ & 2613,0 \\
\hline $\begin{array}{l}\text { Morro do Massena / } \\
\text { Massena Mount }\end{array}$ & Minas Gerais/Rio de Janeiro & $\begin{array}{l}\text { Serra do Itatiaia/ } \\
\text { Itatiaia Range }\end{array}$ & 2603,0 \\
\hline $\begin{array}{l}\text { Pico da Cabeça de Touro / } \\
\text { Cabeça de Touro Peak }\end{array}$ & São Paulo & $\begin{array}{l}\text { Serra Fina } \\
\text { Fina Range }\end{array}$ & 2600,0 \\
\hline
\end{tabular}

Fonte/Source: IBGE, Diretoria de Geociências, Coordenação de Cartografia, Cadastro de Pontos mais Altos do Brasil e Coordenação de Geodésia, Projeto Pontos Culminantes.

Nota: Foram considerados os pontos com altitude superior a 2600 metros. / Note: Only the points over 2 600 meters were included.

(1) Altitudes obtidas a partir da aplicação da versão 2015 do modelo de ondulação geoidal (MAPGEO 2015). (2) Fronteira com a Venezuela. (3) Fronteira com a Guiana. (4) Base Cartográfica Vetorial Contínua do Estado do Rio de Janeiro - 1:25 000. / (1) Altitudes obtained through the 2015 version of the geoid undulation model (MAPGEO 2015). (2) Venezuela border. (3) Guyana border. (4) Continuous Vectorial Cartographic Base of Rio de Janeiro state - 1:25000 


\section{Tabela 1.5 - Localização geográfica dos Municípios das Capitais e distância a Brasília - 2019}

Table 1.5 -Geographic location of the Municipalities of the Capital and distance to Brasília - 2019

\begin{tabular}{|c|c|c|c|c|}
\hline \multirow{2}{*}{$\begin{array}{c}\text { Municípios das Capitais/ } \\
\text { Municipalities of } \\
\text { the capital }\end{array}$} & \multicolumn{2}{|c|}{$\begin{array}{l}\text { Localização geográfica/ } \\
\text { Geographic location }\end{array}$} & \multicolumn{2}{|c|}{$\begin{array}{l}\text { Distância a Brasília }(\mathrm{km}) / \\
\text { Distance to Brasília }(\mathrm{Km})\end{array}$} \\
\hline & $\begin{array}{l}\text { Latitude/ } \\
\text { Latitude }\end{array}$ & $\begin{array}{l}\text { Longitude/ } \\
\text { Longitude }\end{array}$ & $\begin{array}{c}\text { Em reta (1)/ } \\
\text { Straight line (1) }\end{array}$ & $\begin{array}{c}\text { Rodoviária (2)/ } \\
\text { Road (2) }\end{array}$ \\
\hline Porto Velho (RO) & $-8^{\circ} 45^{\prime} 58^{\prime \prime}$ & $-63^{\circ} 54^{\prime} 34^{\prime \prime}$ & 1904,002 & 2589 \\
\hline Rio Branco (AC) & $-9 \circ 58 ' 21 "$ & $-67^{\circ} 48^{\prime} 36^{\prime \prime}$ & 2250,489 & 3123 \\
\hline Manaus (AM) & $-3 \circ 8^{\prime} 10^{\prime \prime}$ & $-60^{\circ} 1 ' 32^{\prime \prime}$ & 1933,07 & 3490 \\
\hline Boa Vista (RR) & $2^{\circ} 49^{\prime} 15^{\prime \prime}$ & $-60^{\circ} 40^{\prime} 21^{\prime \prime}$ & 2501,02 & 4275 \\
\hline Belém (PA) & $-1^{\circ} 27^{\prime} 19^{\prime \prime}$ & $-48^{\circ} 30^{\prime} 19^{\prime \prime}$ & 1595,799 & 2120 \\
\hline Macapá (AP) & $0^{\circ} 2^{\prime} 12^{\prime \prime}$ & $-51^{\circ} 3^{\prime} 6^{\prime \prime}$ & 1793,730 & $\ldots$ \\
\hline Palmas (TO) & $-10^{\circ} 11^{\prime} 4^{\prime \prime}$ & $-48^{\circ} 20^{\prime} 1 "$ & 624,400 & 973 \\
\hline São Luís (MA) & $-2^{\circ} 31^{\prime} 55^{\prime \prime}$ & $-44^{\circ} 17^{\prime} 52^{\prime \prime}$ & 1527,526 & 2157 \\
\hline Teresina (PI) & $-5^{\circ} 5^{\prime} 26^{\prime \prime}$ & $-42^{\circ} 49^{\prime} 2^{\prime \prime}$ & 1314,589 & 1789 \\
\hline Fortaleza (CE) & $-3^{\circ} 43^{\prime} 27^{\prime \prime}$ & $-38 \circ 31 ' 31^{\prime \prime}$ & 1691,499 & 2378 \\
\hline Natal (RN) & $-5^{\circ} 47^{\prime} 4^{\prime \prime}$ & $-35^{\circ} 12^{\prime} 29^{\prime \prime}$ & 1779,777 & 2422 \\
\hline João Pessoa (PB) & $-7 \circ 6 ' 55^{\prime \prime}$ & $-34^{\circ} 53^{\prime} 24^{\prime \prime}$ & 1717,457 & 2245 \\
\hline Recife (PE) & $-8^{\circ} 3^{\prime} 46^{\prime \prime}$ & $-34^{\circ} 53^{\prime} 20^{\prime \prime}$ & 1658,798 & 2220 \\
\hline Maceió (AL) & $-9 \circ 39^{\prime} 37^{\prime \prime}$ & $-35^{\circ} 44^{\prime} 22^{\prime \prime}$ & 1487,959 & 1928 \\
\hline Aracaju (SE) & $-10^{\circ} 54^{\prime} 48^{\prime \prime}$ & $-37 \circ 3^{\prime} 5^{\prime \prime}$ & 1296,379 & 1652 \\
\hline Salvador (BA) & $-12^{\circ} 58^{\prime} 27^{\prime \prime}$ & $-38^{\circ} 30^{\prime} 44^{\prime \prime}$ & 1062,295 & 1446 \\
\hline Belo Horizonte (MG) & $-19^{\circ} 55^{\prime} 25^{\prime \prime}$ & $-43^{\circ} 56^{\prime} 11^{\prime \prime}$ & 625,869 & 716 \\
\hline Vitória (ES) & $-20^{\circ} 19^{\prime} 16^{\prime \prime}$ & $-40^{\circ} 20^{\prime} 21^{\prime \prime}$ & 948,925 & 1238 \\
\hline Rio de Janeiro (RJ) & $-22^{\circ} 54^{\prime} 10^{\prime \prime}$ & $-43^{\circ} 10^{\prime} 26^{\prime \prime}$ & 936,888 & 1148 \\
\hline São Paulo (SP) & $-23^{\circ} 33^{\prime} 5^{\prime \prime}$ & $-46^{\circ} 38^{\prime} 4^{\prime \prime}$ & 875,646 & 1015 \\
\hline Curitiba (PR) & $-25^{\circ} 25^{\prime} 2^{\prime \prime}$ & $-49^{\circ} 16^{\prime} 7^{\prime \prime}$ & 1081,759 & 1366 \\
\hline Florianópolis (SC) & $-27^{\circ} 35^{\prime} 51^{\prime \prime}$ & $-48^{\circ} 32^{\prime} 59^{\prime \prime}$ & 1317,011 & 1673 \\
\hline Porto Alegre (RS) & $-30^{\circ} 1^{\prime} 48^{\prime \prime}$ & $-51^{\circ} 13^{\prime} 43^{\prime \prime}$ & 1621,605 & 2027 \\
\hline Campo Grande (MS) & $-20^{\circ} 27^{\prime} 45^{\prime \prime}$ & $-54^{\circ} 36^{\prime} 31^{\prime \prime}$ & 877,744 & 1134 \\
\hline Cuiabá (MT) & -15०36'2" & $-56^{\circ} 6^{\prime} 1^{\prime \prime}$ & 875,725 & 1133 \\
\hline Goiânia (GO) & $-16^{\circ} 40^{\prime} 49^{\prime \prime}$ & $-49^{\circ} 15^{\prime} 23^{\prime \prime}$ & 173,581 & 209 \\
\hline Brasília (DF) & $-15^{\circ} 47^{\prime} 4^{\prime \prime}$ & $-47^{\circ} 54^{\prime} 29^{\prime \prime}$ & - & - \\
\hline
\end{tabular}

Fonte/Source : IBGE, Diretoria de Geociências, Coordenação de Estruturas Territoriais.

(1) Calculada com base nas coordenadas planimétricas das Sedes Municipais, 2018.

(2) Dados do Departamento Nacional de Infraestrutura de Transportes - DNIT.

(1) Straight-line distance calculated based on the planimetric coordinates of the Municipal Headquarters, 2018

(2) Data from the National Department of Transportation Infrastructure-DNIT. 